\title{
A case of immune-mediated haemolytic anaemia secondary to a gastro-intestinal stromal tumour in a dog.
}

Schiavo L., Dobson JM, McCallum K.

1Queen's Veterinary School Hospital, University of Cambridge, Veterinary School

Signalment: 9 years 8 months, female neutered, Cocker Spaniel.

\section{Presenting signs:}

.Acute onset of lethargy.

-Pale mucosal membranes.

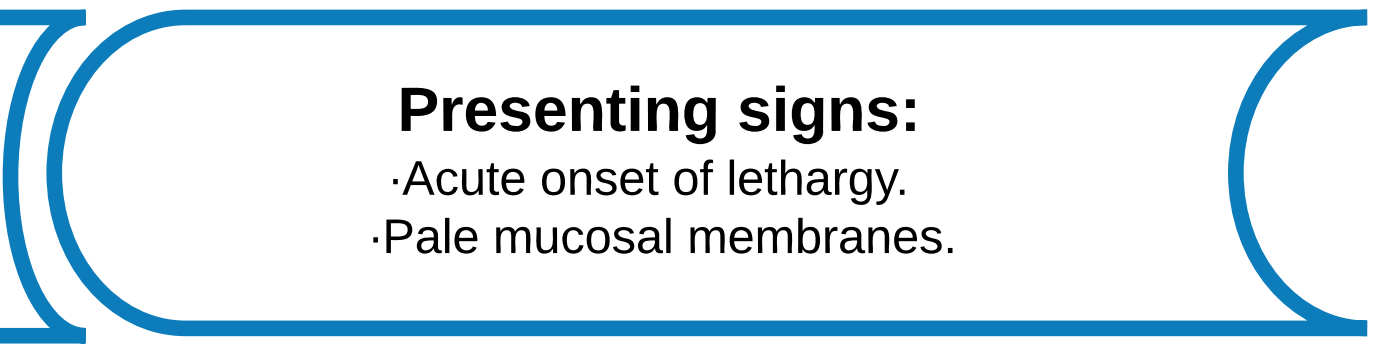

\section{Diagnostic findings:}

-Moderate, regenerative anaemia with evidence of spherocytosis and agglutination.

Coombs' Test positive.

Abdominal ultrasound: $1.8 \mathrm{~cm} \times 3.6$ $\mathrm{cm}$ hypoechoic caecal mass.

Unremarkable thoracic radiographs,

-Idexx SNAP 4Dx negative.

.Urinalysis: specific gravity 1.046; bilirubinuria.

-Cytology of the mass compatible with a mesenchymal neoplasia.
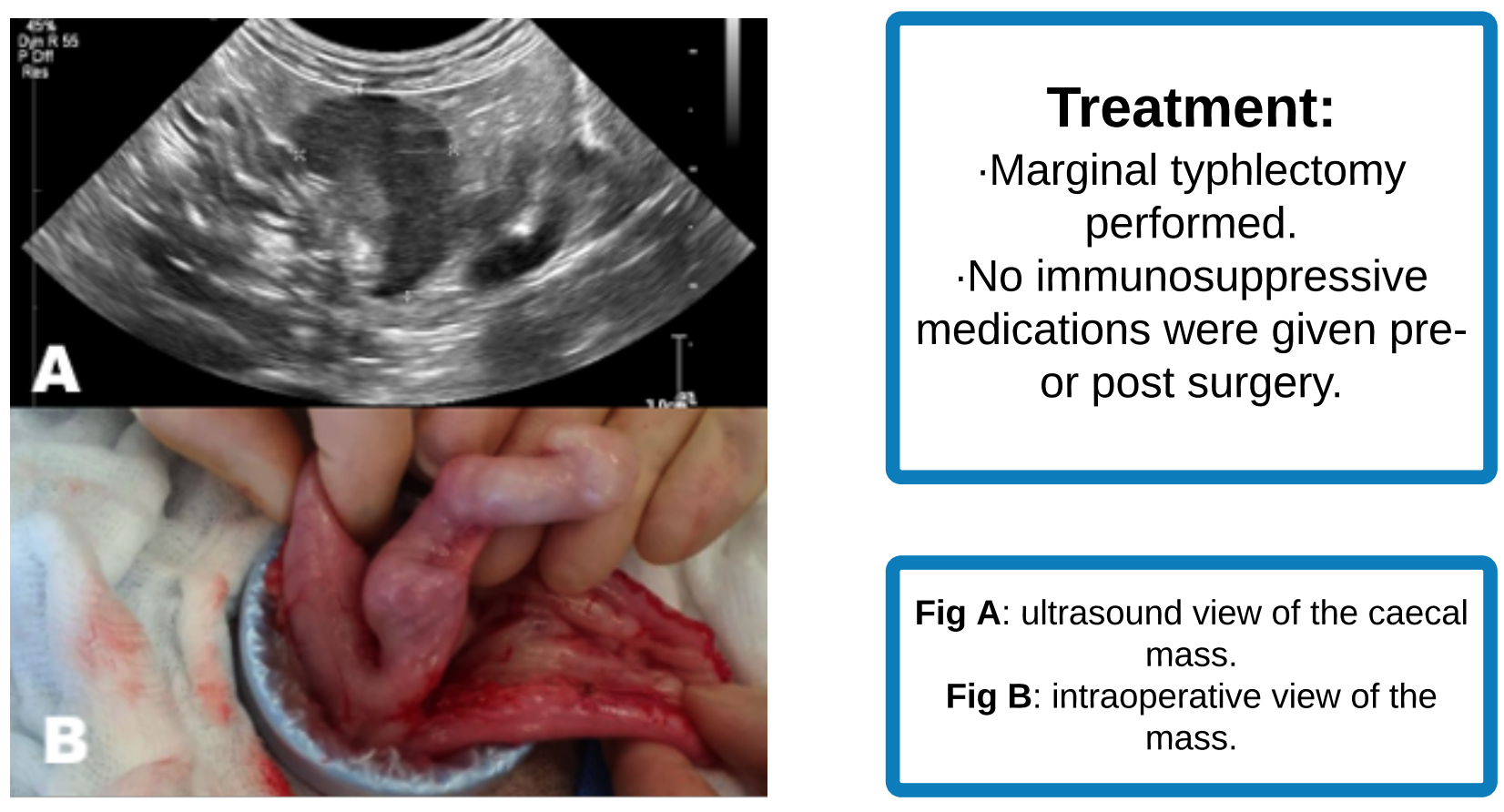

Fig A: ultrasound view of the caecal mass.

Fig B: intraoperative view of the mass.

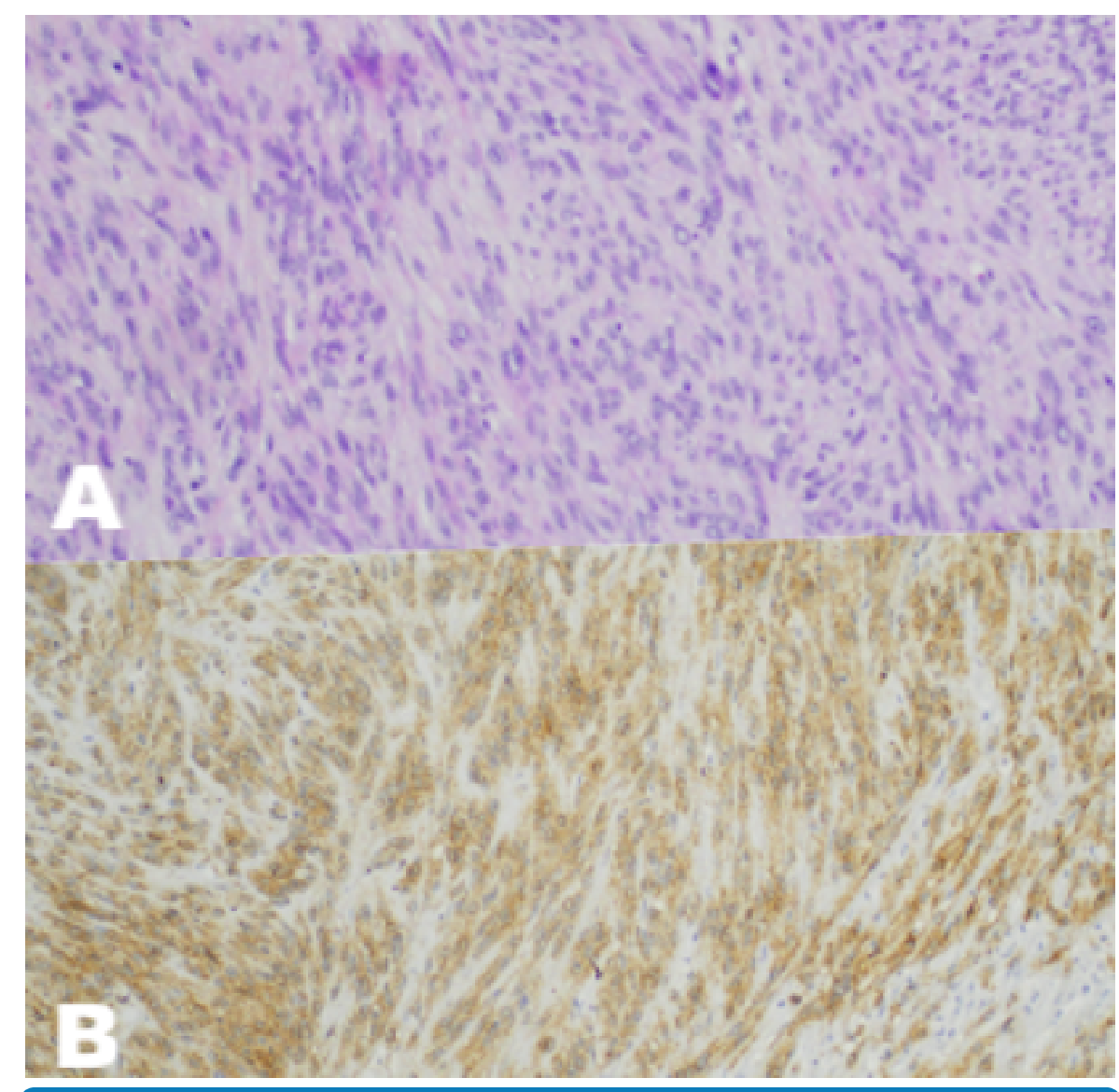

Fig A haematoxylin and eosin staining of the mass. Fig B CD 117 (c-Kit)staining of the mass.
Histopathology: The neoplastic cells are arranged in bundles, interlacing streams and herring-bone patterns, with occasional nuclear palisading, and are supported by a moderate amount of fibrous stroma. The individual neoplastic cells are of moderate size, spindle-shaped, with indistinct cell borders and scant eosinophilic, mildly vacuolated cytoplasm. The nuclei are oval to round, centrally located with finely stippled to vesicular chromatin and up to two, small, eosinophilic, variably distinct nucleoli.

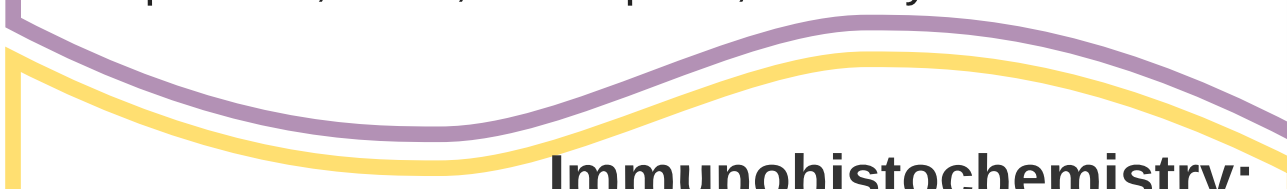

\section{Immunohistochemistry:}

Diffusely approximately $60-70 \%$ of the neoplastic cells exhibit a weak to moderately strong, punctuate, predominantly membranous but also cytoplasmic staining for CD117 (c-kit).

Diffusely approximately $40 \%$ of the neoplastic cells exhibit a weak cytoplasmic staining for smooth muscle actin

(SMA).

\section{Outcome:}

The patient was diagnosed with immune-mediated haemolytic anaemia (IMHA) secondary to a gastrointestinal stromal tumour (GIST). Four weeks after surgical removal the anaemia had resolved. Coombs' testing was negative and no evidence of spherocytosis was present in the blood smear. The patient is reported to be doing well 8 months after initial presentation.

IMHA is a well-reported paraneoplastic syndrome, particularly in association with lymphoproliferative disorders. The condition has previously been reported in humans with solid tumours and, as far as the authors are aware, this case represents the first report of IMHA developing secondary to a GIST in the veterinary literature. 